\title{
A Kind of Nonlinear Feedback Control and Its Application
}

\author{
X. K. Zhang \\ Navigation College \\ Dalian Maritime University \\ Dalian, Liaoning, China
}

\author{
G. Q. Zhang \\ Navigation College \\ Dalian Maritime University \\ Dalian, Liaoning, China
}

\begin{abstract}
In order to improve the control performance of feedback control, this note presents a novel concept "nonlinear feedback control". Different from the conventional linear feedback control, a nonlinear function of error signal is fed back to the controller. The controller is still designed by virtue of the linear or nonlinear control algorithm. The outstanding advantage of the nonlinear feedback control lies in that it requires less control energy and could obtain better control effect. Finally, two simulation experiments have been included to illustrate the effectiveness of the novel concept.
\end{abstract}

Keywords-nonlinear feedback; closed-loop gain shaping; robust control

\section{INTRODUCTION}

The rapid development of control theory and technology is an important symbol of modern science and civilization. The development of control theory has caused great changes in the development of society and economy, which influences the daily life of people every hour and moment. For example, the launch of spacecraft is a typical control task, as well as some other industrial processes [1]. Once the installation of the facility of a chemical plant or a power plant has been completed, the remaining production process can be regarded as a problem of automatic control. Nearly everything will be out of order without the influence of automatic control, which has penetrated into all fields of social development and becomes indispensable.

Automatic control means that in the absence of the direct involvement of people, any quantity of interest (namely the controlled variable) in machinery, mechanism or industrial process (the controlled plant) is maintained or altered in accordance with a desired manner by utilizing an additional equipment or device (control device or controller). For instance, the temperature can be maintained in the refrigerator, marine ships automatically navigate according to a preset course or intended track. The automatic control has developed for more than 200 years, since the advent of the first automatic control system in 1788. Although its foundation and development result from the needs of industrial production, its application has been extended to every aspect of the human society [2-7].

In order to control the plant to follow the predetermined reference, the control law is normally designed on the basis of the error signal. If the output deviates from the desired input, accordingly the controller increase or decrease the control action. The system output is a feedback signal corresponding to the reference one. Thus, one calls it as feedback control. Feedback control is the most basic control strategy. To the best knowledge of the authors, the conventional error based feedback control is one of the most widely used control method. In recent 20 years, the theoretical algorithm of controller design has undergone a transitional period from linear control theory into nonlinear control theory, just to improve the control performance.

This is a nonlinear control development age. Although our faith in many of the linear controls in which our forerunners fervently believed has weakened, our confidence in the linear feedback control properties remains the same as theirs. This modern faith in linear feedback control is proved by the fact that papers annually published in all kinds of journals are mounting to astronomical figures and shows no signs of ceasing to rise at present. However, there are few literatures about nonlinear feedback control through searching the Web of Knowledge. Even so, nonlinear feedback control, a new life in the field of control theory and technology, is in the bud. The origin of its thought lies in that the control effect is increased to reduce the output error, when the output error becomes bigger, the control effect is decreased to reduce the output error, when the output error becomes smaller. However, the relationship between the control law and the error signal is not a simple linear relationship but some kind of a nonlinear one. The nonlinear feedback control may provide a better control performance. Figure 1 shows the graph of the development of automatic control. A variety of nonlinear control theory and methods that currently proposed all belong to the category of linear feedback control, while the birth of the nonlinear feedback control will provide new opportunities for the enhancement of the performance of automatic regulating control.

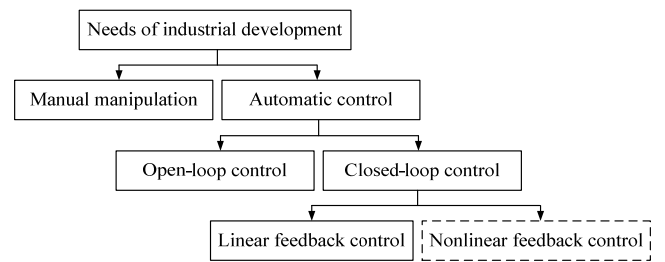

FIGURE I .THE GRAPH OF THE DEVELOPMENT OF AUTOMATIC CONTROL. 


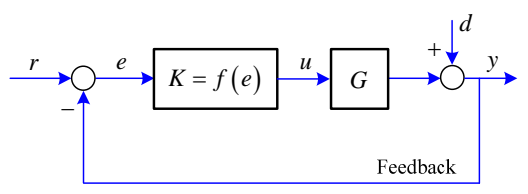

FIGURE II .THE STANDARD FEEDBACK CONTROL BLOCK DIAGRAM.

Standard feedback control block diagram is as shown in Fig.2, where $r$ is the reference, $y$ is the system output, $e=r-y$ is the error signal, $K=f(e)$ is the nonlinear control law, $u$ is the controller output, $G$ is the plant, $d$ is the disturbance. The nonlinear control law of linear feedback control is $u=f(e) \cdot e$; while the nonlinear control law of nonlinear feedback control is designed as $u=f(e) \cdot g(e)$, where $g(e)$ is a nonlinear function of feedback error. The difference between $u=f(e) \cdot g(e)$ and $u=f(e) \cdot e$ is using nonlinear feedback function $g(e)$ to substitute linear feedback $e$ with the control law $f(e)$ remaining unchanged, whose objective is to obtain the same control effect with less control energy or better control performance.

Theoretically, $u=f(e) \cdot g(e)$ could be transformed into $u=h(e) \cdot e$. However, sometimes the deduction of $h(e)$ may be very complex and difficult to obtain. The design philosophy of nonlinear feedback control is under the premise of that $f(e)$ or $K$ has been determined, then the linear feedback variable $e$ is substituted by a nonlinear feedback driving function $g(e)$ through selection, which can save a lot of control energy. This can avoid the deduction of $h(e)$, and also improves the performance of the original control law at the same time. Nonlinear feedback is also existent in the real life. For example, the human brain can beautify the image of a person in the mirror through local non-linearization; Human perception of things around can be affected by individual mood, weather and other factors, which also is a typical case of nonlinear feedback.

Brief theoretical analysis is presented to further demonstrate the advantages of nonlinear feedback control. The nonlinear feedback function $g(e)$ is selected as $\operatorname{Asign}(r-y)|r-y|^{0.6} \quad$ here, according to $(1+x)^{m}=1+m x+(m(m-1) / 2 !) x^{2}+\cdots+(m(m-1) \cdots(m-n+1) / n !) x^{n}+\cdots$, wherein $-1<x<1,1+x$ is substituted by $r-y$; if $m=0.6$ and it is kept to the third order, then it can be proved that $-1.52<\operatorname{sign}(r-y)|r-y|^{0.6}<1.52$ when $|r-y|<2$. Furthermore, $-1 \leq A \operatorname{sign}(r-y)|r-y|^{0.6} \leq 1$, when $|r-y|<2$ and $|A|<0.66$. It can be obtained that the function of $-1 \leq A \operatorname{sign}(r-y)|r-y|^{0.6} \leq 1$ is same as that of $\sin (\omega(r-y))$. Reference [8] points out that sine function is another available function of nonlinear feedback, and theoretically proves that the nonlinear feedback driven by sine function can effectively reduce the output energy of controller on the premise of not changing the original controller and the control effect.

\section{SIMULATION EXPERIMENTS}

This paper presents two examples to illustrate the improvements or effectiveness of nonlinear feedback control strategy. In example 1, the training vessel "Yulong" is selected as the plant and the nonlinear cancellation part of nonlinear PD controller [3] is ignored firstly, which is designed using the Backstepping method, only the linear PD controller is used when the nonlinear feedback configuration is considered. Then the power function $g(r-y)=0.2 \operatorname{sign}(r-y)|r-y|^{0.6} \quad$ is utilized to construct the nonlinear feedback. The reference course is set to $50^{\circ}$ and the mathematical models for ship course keeping control is $\ddot{\psi}=-K / T\left(\alpha \dot{\psi}+\beta \dot{\psi}^{3}\right)+(K / T) \delta$, with ship model parameters $K=0.48 \mathrm{~s}-1, \quad T=216.58 \mathrm{~s}, \quad \alpha=9.16$, $\beta=10814.30 \quad$; PD controller parameters $k_{p}=5.51, k_{d}=150.4$ [9-13]. Fig.3 shows the logic diagram of system simulation test, wherein $g(r-y)=r-y$ when it is linear feedback.

Simulation diagram of Simulink for ship course keeping control is shown in Fig.4. Rudder servo system is also considered in the simulation, the steering engine is modeled as a system with single hydraulic circuit analog control variable $[8,9]$, the maximum rudder ratio is $\pm 5 \%$ s and the saturation rudder angle is $\pm 35^{\circ}$.

When the ship is navigating on the sea, the sway motion and heading deviation are caused mainly by wind and wave disturbance, therefore the effects of wind and wave can not be neglected in the simulation. For the wind disturbance, it is divided into the average wind and impulse wind. The impulse wind is implemented using white noise while the average wind is related with the leeway and is expressed as an equivalent rudder angle $\delta_{\text {wind }}$. According to [8], $\delta_{\text {wind }}$ can be computed by an empirical formula shown in Eq. (1).

$$
\delta_{\text {wind }}=K^{0}\left(\frac{V_{\mathrm{R}}}{V}\right)^{2} \sin \gamma
$$

Where $K^{0}$ is the coefficient of leeway, $V_{\mathrm{R}}$ the relative wind speed to the ship, $V$ the ship speed, $\gamma$ the wind angle on the bow. When the wind scale is Beaufort No.6 and the wind angle on the bow is $-30^{\circ}$, the equivalent rudder angle of wind can be calculated out as $\delta_{\text {wind }}=3^{\circ}$.

For the wave disturbance in the simulation, a simplified model is used which is a second order oscillating system driven by a white noise, and the transfer function of the wave model under the wind scale of Beaufort No.6 is[8]

$$
h(s)=\frac{0.4198 s}{s^{2}+0.3638 s+0.3675}
$$


The white noise with noise power 0.0001 is simulated by sample time of $0.5 \mathrm{~s}$, it is the same as that in the simulation of random wind.

Fig.5 shows the course keeping nonlinear feedback control results of "Yulong". It is very convenient to obtain that average rudder angle of stable course keeping control is $6.1^{\circ}$, the frequency is $3.5 \mathrm{~Hz}$ and the rise time of course keeping control is about 62 seconds for nonlinear feedback control; while the average rudder angle is $6.4^{\circ}$, the frequency is $3.7 \mathrm{~Hz}$ and the rise time of course keeping control is about 80 seconds for linear feedback control. Moreover, the quantity of controlled action in 500 seconds is $10 \%$ reduced by nonlinear feedback control.

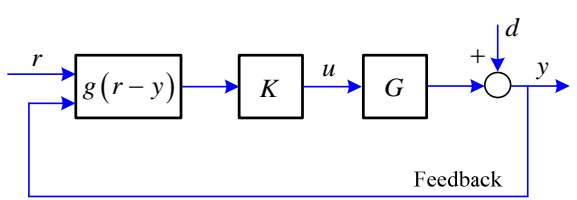

FIGURE III.LOGIC DIAGRAM OF SYSTEM SIMULATION TEST.

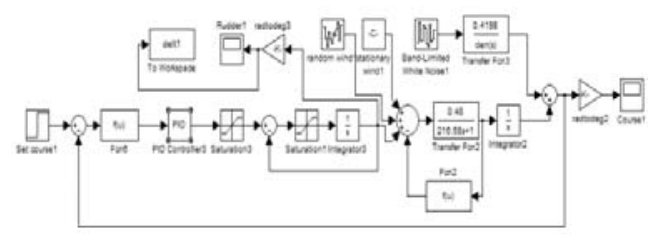

FIGURE IV.SIMULATION DIAGRAM OF SIMULINK FOR SHIP COURSE KEEPING CONTROL
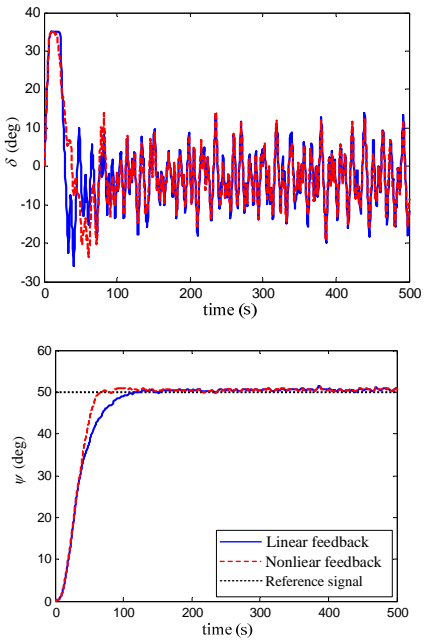

FIGURE V V.COMPARATIVE RESULTS OF SHIP COURSE KEEPING NONLINEAR FEEDBACK CONTROL.

In example 2, a comparison between the power function nonlinear feedback and linear feedback of the PD controllers of water tank liquid level in the literature [14] designed by robust control theory is made. The plants of water tank liquid level control system are composed of a water inlet valve, an outlet valve and a water tank. Please refer to literature [15] for detailed parameters, where the water tank height is $2 \mathrm{~m}$, bottom area is $1 \mathrm{~m} 2$, cross-sectional area of output tube is 0.05 $\mathrm{m} 2$, the initial water level is $0.5 \mathrm{~m}$, the maximum water inflow of water tank is $0.5 \mathrm{~m} 3 / \mathrm{s}$.

The transfer function, i.e. the nominal mathematical model, between the water level and the water inflow can be expressed by Eq.(2), which is utilized for the controller design. The reference signal of water level system is a square wave ranged from 0.5 to $1.5 \mathrm{~m}$, with the frequency of $0.1 \mathrm{rad} / \mathrm{s}$. Therefore, the water level varies from $0.5 \mathrm{~m}$ to $1.5 \mathrm{~m}$ in a cycle of $31.4 \mathrm{~s}$. If the crossover frequency $1 / T_{1}$ equals $1 \mathrm{rad} / \mathrm{s}$, then the parameters of the robust PD controller can be obtained by utilizing closed-loop gain shaping algorithm [10], wherein $K_{p}=1.25, K_{d}=2.5$. In example 2, the driven function of nonlinear feedback $g(r-y)=0.6 \operatorname{sign}(r-y)|r-y|^{0.6}$ is adopted.

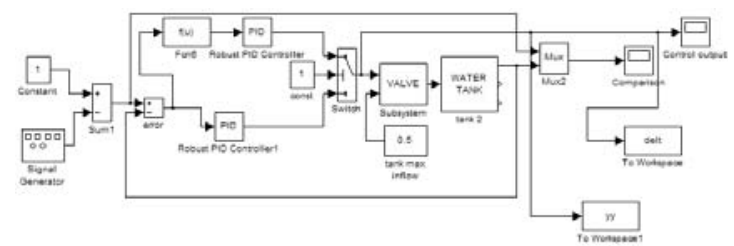

FIGURE VI.SIMULATION DIAGRAM OF SIMULINK FOR WATER TANK CONTROL
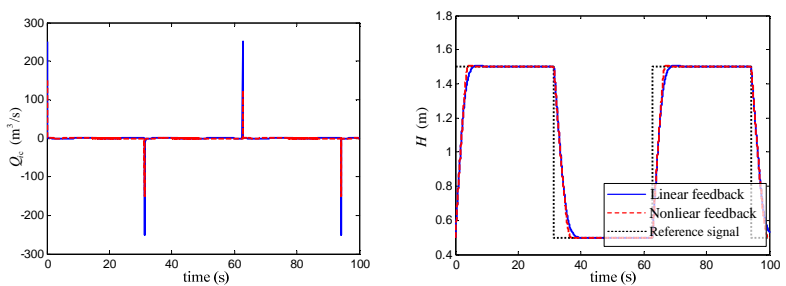

FIGURE VII.COMPARISON RESULTS OF TANK LIQUID LEVEL CONTROL SYSTEM.

Simulation diagram of Simulink for water tank control is shown in Fig.6. Fig.7 shows the comparison results of example 2 by using nonlinear feedback control and linear feedback control, where attention should be paid to that the ordinate $Q_{i c}$ of Fig.7 which represents the control command variables given by two control strategies, while the actual control input $Q_{i}$ after adding limited treatment can satisfy the requirement of maximum water inflow. It can be seen from Fig.7 that the average amount of water inflow under the control of nonlinear feedback is $0.56 \mathrm{~m} 3 / \mathrm{s}$, and the maximum absolute value is 150.8; however, the average amount of water inflow under the control of linear feedback is $0.98 \mathrm{~m} 3 / \mathrm{s}$, and the maximum absolute value is 251.25. In addition, the performance of the nonlinear feedback control on water level is better than that of linear feedback control, where the rise time is shortened about 2 s.

$$
G(s)=\frac{H(s)}{Q_{i}(s)}=\frac{0.8}{s(2 s+1)}
$$

\section{CONCLUSION}

In this paper, the proposed nonlinear feedback control will become another landmark of the development of control 
theory and will further promote the rapid development of control theory and technology.

\section{ACKNOWLEDGMENTS}

The authors would like to thank anonymous reviewers for their valuable comments to improve the quality of this note. This work is partially supported by the National Natural Science Foundation of China (Grant No. 51109020, 50979009), and the Fundamental Research Funds for the Central University (Grant No. 2014YB01, 3132014302).

\section{REFERENCES}

[1] Iasson Karafyllis, Zhongping Jiang. Stability and Stabilization of Nonlinear Systems. Springer-Verlag, London, (2011).

[2] I.J.Nagrath, M.Gopal. Control systems engineering. John Wiley \& Sons, Inc., New York, (1981).

[3] Xianku Zhang. Ship motion concise robust control. Beijing: Science press, (2012).

[4] G M.Dovgobrod. Development of an adaptive algorithm for ship motion control on a nonlinear path. Gyroscopy and Navigation 3(1), 41-46 (2012).

[5] Harl Nathan1, Balakrishnan S N. Impact time and angle guidance with sliding mode control. IEEE Transactions on Control Systems Technology 20(6), 1436-1449 (2012).

[6] Hicham El bahja, Pastora Vega Cruz, Othman Bakka. Nonlinear feedback control of a nutrient removal biological plant. The 20th Mediterranean Conference on Control and Automation, Barcelona, Spain, 1415-1420 (2012).

[7] Nicholas Fischer, Kan Zhen, Rushikesh Kamalapurkar, et al. Saturated RISE feedback control for a class of second-order nonlinear system. IEEE Transaction on Automatic Control 59(4), 1094-1099 (2014).

[8] Xianku Zhang. Control algorithm for autopilot driven by sine of course deviation. Navigation of China 34(1), 1-4 (2011).

[9] Xianku Zhang, Yicheng Jin. Control system modeling and numerical simulation(second edition). Dalian: Dalian maritime university press (2013).

[10] Xianku Zhang, Xinle Jia. Simplification of $\mathrm{H} \infty$ mixed sensitivity algorithm and its application. Automatic Control and Computer Sciences 36(3), 28-33 (2002).

[11] Xianku Zhang, Guoqing Zhang. Stabilization of pure unstable delay systems by the mirror mapping technique. Journal of Process Control 23(10), 1465-1470 (2013).

[12] Guoqing Zhang, Xianku Zhang. Concise robust adaptive path-following control of underactuated ships using DSC and MLP. IEEE Transaction on Oceanic Engineering, to appear (2014).

[13] Xianku Zhang, Guoqing Zhang. Researches on Williamson Turn for Very Large Carriers. Naval Engineers Journal 124(4), 113-119 (2013).

[14] Xianku Zhang, Xinle Jia. A new method for solving PID Parameters. Systems Engineering and Electronics 22(8), 4-5 (2000).

[15] Roger Jang. Fuzzy Toolbox in Matlab5.2 Manuel. The Math Works, Inc. (1998). 